\title{
Clinical and Radiographic Comparison of Influenza Virus-associated Pneumonia among Three Viral Subtypes
}

\author{
Takashi Ishiguro ${ }^{1}$, Noboru Takayanagi ${ }^{1}$, Tetsu Kanauchi ${ }^{2}$, Ryuji Uozumi ${ }^{3}$, Eriko Kawate ${ }^{1}$, \\ Yotaro Takaku ${ }^{1}$, Naho Kagiyama ${ }^{1}$, Yoshihiko Shimizu ${ }^{4}$, Toshiko Hoshi ${ }^{2}$, \\ Satoshi Morita ${ }^{3}$ and Yutaka Sugita ${ }^{1}$
}

\begin{abstract}
Objective Presently, the predominant subtypes of influenza viruses in the world, except for those in local epidemics, include influenza pandemic H1N1 2009 (pH1N1), H3N2, and B viruses. There are few reports on the differences in the clinical features, radiographic findings, treatment, and outcomes of influenza virusassociated pneumonia among these three viral subtypes. The purpose of this study was to investigate whether the clinical features, radiographic findings, treatment, and outcomes differ among the viral subtypes.

Methods We retrospectively analyzed 96 patients with influenza virus-associated pneumonia whose viral subtypes were clarified.

Results Patients with pH1N1 virus-associated pneumonia tended to be young. The frequency of primary viral pneumonia differed among the virus-associated pneumonia subtypes $(\mathrm{pH} 1 \mathrm{~N} 1,80 \%$; $\mathrm{H} 3 \mathrm{~N} 2,26.5 \%$; and $\mathrm{B}$, $31 \%$ ). Patients with pH1N1 virus-associated pneumonia more frequently showed bilateral ground-glass opacities (GGOs), which affected more lobes than in patients with H3N2 and B virus-associated pneumonia. However, patients with $\mathrm{H} 3 \mathrm{~N} 2$ virus-associated pneumonia showed a higher frequency of consolidation and diffuse bronchial wall thickening than did the patients with $\mathrm{pH} 1 \mathrm{~N} 1$ virus-associated pneumonia. The severity and mortality did not differ among the three pneumonia subtypes.

Conclusion In the patients who developed influenza virus-associated pneumonia, those with pH1N1 virusassociated pneumonia frequently developed primary viral pneumonia resulting in bilateral and broad areas of GGOs on imaging, whereas patients with H3N2 virus-associated pneumonia frequently showed consolidation and diffuse bronchial wall thickening on pulmonary imaging.
\end{abstract}

Key words: influenza, pneumonia, subtypes, computed tomography

(Intern Med 55: 731-737, 2016)

(DOI: 10.2169/internalmedicine.55.5227)

\section{Introduction}

Influenza viruses cause significant morbidity and mortality globally that result in severe illness in 3-5 million people and death in up to 500,000 individuals during epidemic years (1). The pandemic influenza in 2009 strongly affected clinical practice. Pneumonia is the leading complication of influenza virus infection, and many cases of pandemic influ- enza H1N1 2009 (pH1N1) virus-associated pneumonia (VAP) have been reported.

Presently, the predominant subtypes in the world, except for those in local epidemics, include influenza pH1N1, H3N2, and B viruses. There are few reports on the differences in the clinical features, radiographic findings, treatment, and outcomes of influenza VAP among the pH1N1, $\mathrm{H} 3 \mathrm{~N} 2$, and B viral subtypes (2). Therefore, the purpose of this study was to investigate whether the clinical features,

\footnotetext{
${ }^{1}$ Department of Respiratory Medicine, Saitama Cardiovascular and Respiratory Center, Japan, ${ }^{2}$ Department of Radiology, Saitama Cardiovascular and Respiratory Center, Japan, ${ }^{3}$ Department of Biomedical Statistics and Bioinformatics, Kyoto University Graduate School of Medicine, Japan and ${ }^{4}$ Department of Pathology, Saitama Cardiovascular and Respiratory Center, Japan Received for publication February 24, 2015; Accepted for publication June 28, 2015 Correspondence to Dr. Takashi Ishiguro, ishiguro.takashi@pref.saitama.lg.jp
} 
radiographic findings, treatment, and outcomes differ among these three viral subtypes.

\section{Materials and Methods}

We conducted a retrospective study of all patients hospitalized with pneumonia over a 12-year period from January 2002 through March 2014 at our institution in Saitama, Japan. Excluded patients comprised those showing immunosuppression (AIDS or receiving chemotherapy), nursing home residents, and those with tuberculosis, non-resected lung cancer, or a confirmed alternative diagnosis lasting until the end of the follow-up. The diagnosis of causative microorganisms was made according to the results of a semiquantitative culture of respiratory samples or blood, paired sera, and urinary antigen tests for Streptococcus pneumoniae and Legionella pneumophila, as reported previously (3).

The types of influenza-associated pneumonia were judged by physicians based on a previous report (4). Severe community-acquired pneumonia was defined when at least one major criterion or three minor criteria of the IDSA/ATS guidelines (5) were present. Computed tomography (CT) scans were independently interpreted by two radiologists, and any disagreement was discussed and resolved by consensus. Diffuse bronchial wall thickening was defined when thickened bronchial walls were present in at least 4 lobes. Lymphadenopathy was defined as a short axis of hilar or mediastinal lymph nodes of $\geq 10 \mathrm{~mm}$. Approval for this study was obtained from the institutional clinical research ethics board (no. 2014006).

\section{Statistical analysis}

The data are presented using descriptive statistics for continuous variables and frequencies for categorical variables. Differences between groups were analyzed with an analysis of variance (ANOVA) for continuous variables and chisquare tests for categorical variables. In all tests, $\mathrm{p}$ values of $<0.05$ were considered to be statistically significant. For CT data, kappa coefficients were calculated to assess the agreement between the two radiologists. All statistical analyses were performed using the SAS version 9.3 (SAS Institute, Cary, USA) software program.

\section{Results}

\section{Patients}

From January 2002 to August 2014, 1,810 patients with pneumonia were admitted to our institution, of whom 194 were diagnosed as having influenza-associated pneumonia based on positive rapid antigen tests, increased antibody titers of paired sera, or positive results of reverse transcription polymerase chain reaction (RT-PCR) analyses. Of these 194 patients, 98 were excluded because we could not differentiate the virus subtype in these patients. These included 9 pa- tients ( 6 before 2009 and 3 after 2009) with a positive rapid antigen test for A viruses in whom serum samples were not obtained in the recovery phase because of their early deterioration to death.

\section{Viral subtypes}

The diagnosis of the viral subtypes was based on the following methods: pH1N1 virus, positive RT-PCR results in $11(55.0 \%)$ and increased antibody titers in $10(50.0 \%)$ of 20 patients; H3N2 virus, positive RT-PCR results in 1 $(2.9 \%)$ and increased antibody titers in $33(97.1 \%)$ of 34 patients; and B virus, positive rapid antigen test in 34 (80.9\%) and increased antibody titers in $12(28.6 \%)$ of 42 patients.

\section{Patient characteristics}

Patients with pH1N1 VAP tended to be younger than those with influenza VAP of the other two viral subtypes $(\mathrm{p}=0.066)$ (Table 1). The frequency of vaccination history differed among patients with each viral subtype infection. Patients with pH1N1 VAP showed higher rates of primary viral pneumonia than those with H3N2 or B VAP (Table 1). Underlying respiratory diseases were present in only $25 \%$ of patients with $\mathrm{pH} 1 \mathrm{~N} 1 \mathrm{VAP}$, and the frequency of underlying respiratory diseases differed among the three VAP groups. C-reactive protein values were significantly lower in the patients with pH1N1 VAP (Table 2). Due to the incomplete workup of the patients' mixed infections, the specific pneumonia type could not be differentiated in $10(10.4 \%)$ patients. Severe cases of pneumonia were present in $25(26 \%)$ of the 97 patients.

\section{Etiology of mixed infection}

The methods used to identify the etiology of pneumonia are shown in Table 3. The most common microorganism in the mixed infection was S. pneumoniae. Other microorganisms included Pseudomonas aeruginosa, Staphylococcus aureus, and Mycoplasma pneumoniae (Table 4).

\section{CT findings}

The kappa values between the two radiologists evaluating the CT images are shown in Table 5. All findings except those for hilar lymphadenopathy showed moderate or good to fair agreement. Patients with pH1N1 VAP showed more frequent bilateral GGOs that affected more lobes than did patients with H3N2 and B VAP (Table 6). However, the frequency of unilateral ground-glass opacities (GGOs) and consolidation were lower in patients with pH1N1 VAP than in patients with $\mathrm{H} 3 \mathrm{~N} 2$ and B VAP. Diffuse bronchial wall thickening was found more frequently in patients with H3N2 VAP. A tree-in-bud appearance was found in 10 patients $(10.4 \%)$, and the frequency of this finding did not differ among the viral subtypes.

\section{Histology}

Specimens were obtained by TBLB from pulmonary lesions corresponding to the GGOs or areas of consolidation 
Table 1. Patient Characteristics.

\begin{tabular}{|c|c|c|c|c|c|}
\hline & $\begin{array}{c}\text { Total } \\
(\mathrm{n}=96)\end{array}$ & $\begin{array}{l}\mathrm{pH} 1 \mathrm{~N} 1 \\
(\mathrm{n}=20)\end{array}$ & $\begin{array}{c}\mathrm{A} / \mathrm{H} 3 \mathrm{~N} 2 \\
(\mathrm{n}=34)\end{array}$ & $\begin{array}{c}\mathrm{B} \\
(\mathrm{n}=42)\end{array}$ & $\mathrm{p}$ value \\
\hline Age, mean (SD) & $66.5(15.7)$ & $59.9(16.4)$ & $70.1(14.5)$ & $66.9(15.6)$ & 0.066 \\
\hline Sex, male & $74(77.1)$ & $16(80.0 \%)$ & $28(82.4 \%)$ & $30(71.4 \%)$ & 0.499 \\
\hline BMI, n & 62 & 16 & 23 & 23 & 0.497 \\
\hline mean $(\mathrm{SD})$ & $22.4(4.90)$ & $23.46(4.80)$ & $22.50(5.05)$ & $21.56(4.88)$ & \\
\hline \multicolumn{6}{|l|}{ Pneumonia type } \\
\hline Primary viral & $38(39.6 \%)$ & $16(80.0 \%)$ & $9(26.5 \%)$ & $13(31.0 \%)$ & 0.002 \\
\hline Mixed viral and bacterial & $32(33.3 \%)$ & $2(10.0 \%)$ & $13(38.2 \%)$ & $17(40.5 \%)$ & \\
\hline Secondary bacterial & $16(16.7 \%)$ & $2(10.0 \%)$ & $9(26.5 \%)$ & $5(11.9 \%)$ & \\
\hline Undifferentiated & $10(10.4 \%)$ & $0(0 \%)$ & $3(8.8 \%)$ & $7(16.7 \%)$ & \\
\hline Vaccination, yes, seasonal & $12(12.5 \%)$ & $2(10.0 \%)$ & $6(17.6 \%)$ & $4(9.5 \%)$ & 0.528 \\
\hline Vaccination, yes, pH1N1 & $6(6.3 \%)$ & $1(5.0 \%)$ & $5(14.7 \%)$ & & 0.030 \\
\hline Underlying pulmonary diseases, yes & $47(49.0 \%)$ & $5(25.0 \%)$ & $21(61.8 \%)$ & $21(50.0 \%)$ & 0.033 \\
\hline COPD & $17(17.7 \%)$ & $1(5.0 \%)$ & $10(29.4 \%)$ & $6(14.3 \%)$ & - \\
\hline Old TB & $6(6.3 \%)$ & & $4(11.8 \%)$ & $2(4.8 \%)$ & - \\
\hline BA & $14(14.6 \%)$ & $3(15.0 \%)$ & $6(17.6 \%)$ & $5(11.9 \%)$ & - \\
\hline Bronchiectasis & $5(5.2 \%)$ & $1(5.0 \%)$ & $1(2.9 \%)$ & $3(7.1 \%)$ & - \\
\hline NTM & $4(4.2 \%)$ & & $1(2.9 \%)$ & $3(7.1 \%)$ & - \\
\hline IP & $7(7.3 \%)$ & & $2(5.9 \%)$ & $5(11.9 \%)$ & - \\
\hline Others & $3(3.1 \%)$ & $1(5.0 \%)$ & & $2(4.8 \%)$ & - \\
\hline Underlying systemic diseases, yes & $40(41.7 \%)$ & $8(40.0 \%)$ & $15(44.1 \%)$ & $17(40.5 \%)$ & 0.936 \\
\hline DM & $18(18.8 \%)$ & $4(20.0 \%)$ & $7(20.6 \%)$ & $7(16.7 \%)$ & 0.898 \\
\hline Post upper digestive system surgery & $8(8.3 \%)$ & & & $1(2.4 \%)$ & - \\
\hline Ischemic heart disease & $8(8.3 \%)$ & $1(5.0 \%)$ & $4(11.8 \%)$ & $3(7.1 \%)$ & - \\
\hline $\mathrm{CHF}$ & $4(4.2 \%)$ & & $3(8.8 \%)$ & $1(2.4 \%)$ & - \\
\hline Cerebrovascular disease & $5(5.2 \%)$ & & $1(2.9 \%)$ & $4(9.5 \%)$ & - \\
\hline Valvular heart disease & $3(3.1 \%)$ & & $3(8.8 \%)$ & & - \\
\hline Arrhythmia & $2(2.1 \%)$ & $1(5.0 \%)$ & $1(2.9 \%)$ & & - \\
\hline Malignancy & $1(1.0 \%)$ & $1(5.0 \%)$ & & & - \\
\hline Psychological disease, dementia & $4(4.2 \%)$ & $1(5.0 \%)$ & $1(2.9 \%)$ & $2(4.8 \%)$ & - \\
\hline Chronic liver disease & $5(5.2 \%)$ & $2(10.0 \%)$ & $2(5.9 \%)$ & $1(2.4 \%)$ & - \\
\hline Neuromuscular disease & $2(2.1 \%)$ & & & $2(4.8 \%)$ & - \\
\hline Connective tissue disease & $2(2.1 \%)$ & & & $2(4.8 \%)$ & - \\
\hline Steroid or immunosuppressant & $2(2.1 \%)$ & & & $2(4.8 \%)$ & 0.269 \\
\hline Smoking history, yes & $58(60.4 \%)$ & $14(70.0 \%)$ & $24(70.6 \%)$ & $20(47.6 \%)$ & 0.075 \\
\hline Severity, severe & $25(26.0 \%)$ & $3(15.0 \%)$ & $12(35.3 \%)$ & $10(23.8 \%)$ & 0.236 \\
\hline Respiratory failure & $49(51.0 \%)$ & $9(45.0 \%)$ & $20(58.8 \%)$ & $20(47.6 \%)$ & 0.523 \\
\hline \multicolumn{6}{|l|}{ Treatment } \\
\hline Preceding antibiotics & $30(31.3 \%)$ & $6(30.0 \%)$ & $8(23.5 \%)$ & $16(38.1 \%)$ & 0.350 \\
\hline Guideline adherence, discordant & $26(27.1 \%)$ & $2(10.0 \%)$ & $10(29.4 \%)$ & $14(33.3 \%)$ & 0.144 \\
\hline Antivirals, yes & $67(69.8 \%)$ & $14(70.0 \%)$ & $19(55.9 \%)$ & $34(81.0 \%)$ & 0.061 \\
\hline$<48 \mathrm{~h}$ & $20(20.8 \%)$ & $1(5.0 \%)$ & $6(17.6 \%)$ & $13(31.0 \%)$ & 0.099 \\
\hline$\geq 48 \mathrm{~h}$ & $47(49.0 \%)$ & $13(65.0 \%)$ & $13(38.2 \%)$ & $21(50.0 \%)$ & \\
\hline Corticosteroids & $21(21.9 \%)$ & $9(45.0 \%)$ & $6(17.6 \%)$ & $6(14.3 \%)$ & 0.018 \\
\hline Mechanical Ventilation & $7(7.3 \%)$ & $2(10.0 \%)$ & $3(8.8 \%)$ & $2(4.8 \%)$ & 0.711 \\
\hline Non-survivors & $4(4.2 \%)$ & & $1(2.9 \%)$ & $3(7.1 \%)$ & 0.381 \\
\hline
\end{tabular}

SD: standard deviation, BMI: body mass index, COPD: chronic obstructive pulmonary disease, TB: tuberculosis, BA: bronchial asthma, NTM: non-tuberculous mycobacteria, IP: interstitial pneumonia, DM: diabetes mellitus, CHF: congestive heart failure

on CT in 8 patients ( 5 with pH1N1 and 3 with H3N2 VAP; 7 with primary viral pneumonia, and 1 with mixed viral and bacterial pneumonia). The cases of three patients were previously reported elsewhere (6-8). The histological findings of survivors with either $\mathrm{pH} 1 \mathrm{~N} 1$ or H3N2 VAP were as follows: in two patients with primary viral pneumonia and mixed viral and bacterial pneumonia, alveoli were not obtained and erosive bronchitis was found. Erosive bronchitis was found in 2 other patients with primary viral pneumonia. In 6 patients whose alveoli could be evaluated, edematous intraseptal thickening, swollen type II cell metaplasia (alveolitis), and intraluminal/airspace organization were found. Fibrin exudation was notable in 3 patients. In one patient with primary pH1N1 viral pneumonia, a diffuse alveolar damage pattern with hyaline membranes and bronchiolitis were also found. In another patient with primary $\mathrm{pH} 1 \mathrm{~N} 1$ viral pneu- monia, hemosiderin-laden macrophages were also detected.

\section{Treatment and outcomes}

Overall, $67(69.8 \%)$ patients received antiviral therapy, of whom $6(6.3 \%)$ required mechanical ventilation and 4 $(4.2 \%)$ died. The frequency of antibiotic treatment discordant with the established guidelines (5) and antiviral use did not differ among the three VAP groups. Patients with pH1N1 VAP received corticosteroid therapy more frequently than those with $\mathrm{H} 3 \mathrm{~N} 2$ and $\mathrm{B}$ virus infection (Table 1). There were no deaths in patients with pH1N1 VAP, and the mortality rate did not differ among the three VAP groups.

\section{Discussion}

In the present study, we focused on influenza VAP that 
Table 2. Laboratory Values.

\begin{tabular}{|c|c|c|c|c|c|}
\hline & & pH1N1 & H3N2 & $\mathrm{B}$ & $n$ value \\
\hline & & $(n=20)$ & $(n=34)$ & $(n=42)$ & $\mathrm{p}$ value \\
\hline \multirow[t]{2}{*}{$\overline{\mathrm{WBC}}$} & $\mathrm{n}$ & 20 & 34 & $\overline{42}$ & 0.075 \\
\hline & $\left(/ \mathrm{mm}^{3}\right)$ & $8,930.0(5,193.9)$ & $10,076.5(4,230.7)$ & $12,423.8(7,572.9)$ & \\
\hline \multirow{2}{*}{ Lymphocytes } & $\mathrm{n}$ & 20 & 34 & 41 & 0.328 \\
\hline & $(\%)$ & $14.31(12.62)$ & $10.53(6.55)$ & $13.15(10.35)$ & \\
\hline \multirow[t]{2}{*}{ Lymphocytes } & $\mathrm{n}$ & 20 & 34 & 41 & 0.189 \\
\hline & $\left(/ \mathrm{mm}^{3}\right)$ & $970.0(865.2)$ & $920.0(581.2)$ & $1,173.7(510.7)$ & \\
\hline \multirow[t]{2}{*}{ Hemoglobin } & $\mathrm{n}$ & 20 & 34 & 39 & 0.425 \\
\hline & $(\mathrm{g} / \mathrm{dL})$ & $13.04(2.49)$ & 12.67 (1.69) & $12.37(1.63)$ & \\
\hline \multirow[t]{2}{*}{ Platelets } & $\mathrm{n}$ & 20 & 34 & 42 & 0.145 \\
\hline & $\left(/ \mathrm{mm}^{3}\right)$ & $22.65(10.73)$ & $19.65(9.41)$ & $23.88(8.53)$ & \\
\hline \multirow[t]{2}{*}{ CRP } & $\mathrm{n}$ & 20 & 34 & 42 & 0.027 \\
\hline & $(\mathrm{mg} / \mathrm{dL})$ & $8.66(7.21)$ & $14.62(10.66)$ & $15.72(9.87)$ & \\
\hline \multirow[t]{2}{*}{ CPK } & $\mathrm{n}$ & 20 & 30 & 38 & 0.388 \\
\hline & (IU/L) & $191.3(161.2)$ & $636.0(1,613.9)$ & $317.8(1,140.0)$ & \\
\hline \multirow[t]{2}{*}{$\mathrm{LDH}$} & $\mathrm{n}$ & 20 & 34 & 42 & 0.165 \\
\hline & (IU/L) & $377.9(219.8)$ & $304.8(113.3)$ & $277.2(229.1)$ & \\
\hline \multirow[t]{2}{*}{$\mathrm{Cr}$} & $\mathrm{n}$ & 20 & 34 & 42 & 0.041 \\
\hline & $(\mathrm{mg} / \mathrm{dL})$ & $0.79(0.22)$ & $1.04(0.54)$ & $0.82(0.36)$ & \\
\hline \multirow[t]{2}{*}{$\mathrm{PaCO}_{2}$} & $\mathrm{n}$ & 20 & 28 & 38 & 0.136 \\
\hline & (Torr) & $33.7(3.56)$ & $41.23(18.95)$ & $38.58(9.77)$ & \\
\hline
\end{tabular}

WBC: white blood cells, CRP: C-reactive protein, CPK: creatine phosphokinase, IU: international unit, $\mathrm{LDH}$ : lactate dehydrogenase, $\mathrm{Cr}$ : creatinine, $\mathrm{PaCO}_{2}$ : partial pressure of arterial carbon dioxide. Values are numbers and means (SD).

Table 3. Diagnostic Methods and Results.

\begin{tabular}{|c|c|c|c|c|c|}
\hline & & Total & pH1N1 & $\mathrm{A} / \mathrm{H} 3 \mathrm{~N} 2$ & $\mathrm{~B}$ \\
\hline & & & $(\mathrm{n}=20)$ & $(n=34)$ & $(n=42)$ \\
\hline \multirow[t]{2}{*}{ Sputum } & $\mathrm{n}$ & $76(79.2 \%)$ & $14(70.0 \%)$ & $30(88.2 \%)$ & $32(76.2 \%)$ \\
\hline & Positive & $14(18.4 \%)$ & $0(0 \%)$ & $9(30.0 \%)$ & $5(15.6 \%)$ \\
\hline \multirow[t]{2}{*}{ Blood culture } & $\mathrm{n}$ & $51(3.1 \%)$ & $11(55.0 \%)$ & $20(58.8 \%)$ & $20(47.6 \%)$ \\
\hline & Positive & $1(2.0 \%)$ & $0(0 \%)$ & $0(0 \%)$ & $1(5.0 \%)$ \\
\hline \multirow[t]{2}{*}{ BALF culture } & $\mathrm{n}$ & $10(10.4 \%)$ & $7(35.0 \%)$ & $2(5.9 \%)$ & $1(2.4 \%)$ \\
\hline & Positive & $0(0 \%)$ & $0(0 \%)$ & $0(0 \%)$ & $0(0 \%)$ \\
\hline \multirow[t]{2}{*}{ Transbronchial aspirate } & $\mathrm{n}$ & $7(7.3 \%)$ & $2(10.0 \%)$ & $4(11.8 \%)$ & $1(2.4 \%)$ \\
\hline & Positive & $3(42.9 \%)$ & $1(50.0 \%)$ & $2(50.0 \%)$ & $0(0 \%)$ \\
\hline \multirow[t]{2}{*}{ Pleural effusion } & $\mathrm{n}$ & $5(5.2 \%)$ & $0(0 \%)$ & $3(8.8 \%)$ & $2(4.8 \%)$ \\
\hline & Positive & $0(0 \%)$ & $0(0 \%)$ & $0(0 \%)$ & $0(0 \%)$ \\
\hline \multirow[t]{2}{*}{ Rapid influenza diagnostic test } & $\mathrm{n}$ & $80(83.3 \%)$ & $17(85.0 \%)$ & $23(67.6 \%)$ & $40(95.2 \%)$ \\
\hline & Positive & $59(73.8 \%)$ & $12(70.6 \%)$ & $14(60.9 \%)$ & $33(82.5 \%)$ \\
\hline \multirow[t]{2}{*}{ PCR } & $\mathrm{n}$ & $12(12.5 \%)$ & $11(55.0 \%)$ & $1(2.9 \%)$ & $0(0 \%)$ \\
\hline & Positive & $12(100 \%)$ & $11(100.0 \%)$ & $1(100.0 \%)$ & $0(0 \%)$ \\
\hline \multirow[t]{2}{*}{ Paired sera } & $\mathrm{n}$ & $87(90.6 \%)$ & $20(100.0 \%)$ & $33(97.1 \%)$ & $34(81.0 \%)$ \\
\hline & Positive & $51(58.6 \%)$ & $10(50.0 \%)$ & $33(100.0 \%)$ & $8(23.5 \%)$ \\
\hline
\end{tabular}

BALF: bronchoalveolar lavage fluid, PCR: polymerase chain reaction

Table 4. Pathogens of Mixed Infection.

\begin{tabular}{lccccc}
\hline & Total & $(\%)$ & $\begin{array}{c}\mathrm{pH} 1 \mathrm{~N} 1 \\
(\mathrm{n}=20)\end{array}$ & $\begin{array}{c}\mathrm{H} 3 \mathrm{~N} 2 \\
(\mathrm{n}=34)\end{array}$ & $\begin{array}{c}\mathrm{B} \\
(\mathrm{n}=42)\end{array}$ \\
\hline Number of mixed infections & 43 & - & 4 & 21 & 18 \\
Streptococcus pneumoniae & 30 & $(69.8)$ & 3 & 13 & 14 \\
Mycoplasma pneumoniae & 2 & $(4.7)$ & 0 & 0 & 2 \\
Haemophilus influenzae & 2 & $(4.7)$ & 0 & 1 & 1 \\
Staphylococcus aureus & 4 & $(9.3)$ & 0 & 3 & 1 \\
Legionella sp. & 1 & $(2.3)$ & 0 & 1 & 0 \\
Chlamydia pneumoniae & 2 & $(4.7)$ & 0 & 1 & 1 \\
GNEB & 3 & $(7.0)$ & 1 & 1 & 1 \\
Pseudomonas aeruginosa & 6 & $(14.0)$ & 0 & 4 & 2 \\
Others & 2 & $(4.7)$ & 0 & 1 & 1 \\
\hline
\end{tabular}

GNEB: gram-negative enteric bacilli
Table 5. Concordance Rate between Radiologists.

\begin{tabular}{lr}
\hline CT findings & kappa values $(95 \% \mathrm{CI})$ \\
\hline GGOs & $0.556(0.311,0.800)$ \\
Crazy-paving pattern & $0.461(0.154,0.769)$ \\
Consolidation & $0.495(0.322,0.668)$ \\
Nodules & $0.548(0.394,0.702)$ \\
Diffuse bronchial wall thickening & $0.527(0.358,0.696)$ \\
Pleural effusion & $0.753(0.610,0.897)$ \\
Unilateral & $0.457(0.186,0.728)$ \\
Bilateral & $0.687(0.498,0.876)$ \\
Mediastinal lymph node swelling & $0.514(0.329,0.698)$ \\
Hilar lymph node swelling & $-0.068(-0.110,-0.027)$ \\
Cardiac enlargement & $0.714(0.529,0.900)$ \\
Pneumothorax & $0.662(0.042,1.000)$ \\
\hline CT: computed tomography, CI: confice
\end{tabular}

CT: computed tomography, CI: confidence interval, GGOs: groundglass opacities. Kappa values: 0-0.4, poor; 0.41-0.60, moderate; 0.61-0.80, good to fair; $0.81-$, excellent. 
Table 6. Characteristics of Computed Tomography Findings.

\begin{tabular}{|c|c|c|c|c|c|c|}
\hline & & $\begin{array}{c}\text { Total } \\
(\mathrm{n}=96)\end{array}$ & $\begin{array}{l}\mathrm{pH} 1 \mathrm{~N} 1 \\
(\mathrm{n}=20)\end{array}$ & $\begin{array}{c}\text { H3N2 } \\
(n=34)\end{array}$ & $\begin{array}{c}\mathrm{B} \\
(\mathrm{n}=42)\end{array}$ & $\mathrm{p}$ value \\
\hline$\overline{\text { GGOs }}$ & Yes & $85(88.5 \%)$ & $19(95.0 \%)$ & $30(88.2 \%)$ & $36(85.7 \%)$ & 0.561 \\
\hline Unilateral & Yes & $32(33.3 \%)$ & $1(5.0 \%)$ & $11(32.4 \%)$ & $20(47.6 \%)$ & 0.004 \\
\hline Bilateral & Yes & $53(55.2 \%)$ & $18(90.0 \%)$ & $19(55.9 \%)$ & $16(38.1 \%)$ & 0.001 \\
\hline Affected lobes & Mean (SD) & $2.75(2.07)$ & $4.25(2.02)$ & $2.79(1.90)$ & $2.00(1.85)$ & $<.001$ \\
\hline Crazy-paving pattern & Yes & $12(12.5 \%)$ & $5(25.0 \%)$ & $3(8.8 \%)$ & $4(9.5 \%)$ & 0.164 \\
\hline Unilateral & Yes & $10(10.4 \%)$ & $3(15.0 \%)$ & $3(8.8 \%)$ & $4(9.5 \%)$ & 0.749 \\
\hline Bilateral & Yes & $2(2.1 \%)$ & $2(10.0 \%)$ & & & 0.021 \\
\hline Affected lobes & Mean (SD) & $0.21(0.78)$ & $0.65(1.57)$ & $0.09(0.29)$ & $0.10(0.30)$ & 0.016 \\
\hline Consolidation & Yes & $76(79.2 \%)$ & $12(60.0 \%)$ & $31(91.2 \%)$ & $33(78.6 \%)$ & 0.024 \\
\hline Unilateral & Yes & $34(35.4 \%)$ & $1(5.0 \%)$ & $14(41.2 \%)$ & $19(45.2 \%)$ & 0.006 \\
\hline Bilateral & Yes & $42(43.8 \%)$ & $11(55.0 \%)$ & $17(50.0 \%)$ & $14(33.3 \%)$ & 0.181 \\
\hline Affected lobes & Mean (SD) & $2.09(1.80)$ & $2.15(2.23)$ & $2.29(1.59)$ & $1.90(1.76)$ & 0.641 \\
\hline Nodules & Yes & $56(58.3 \%)$ & $12(60.0 \%)$ & $22(64.7 \%)$ & $22(52.4 \%)$ & 0.548 \\
\hline Centrilobular nodules & Yes & $54(56.3 \%)$ & $11(55.0 \%)$ & $22(64.7 \%)$ & $21(50.0 \%)$ & 0.493 \\
\hline Tree-in-bud appearance & Yes & $10(10.4 \%)$ & $2(10.0 \%)$ & $3(8.8 \%)$ & $5(11.9 \%)$ & 0.907 \\
\hline Diffuse bronchial wall thickening & Yes & $37(38.5 \%)$ & $2(10.0 \%)$ & $22(64.7 \%)$ & $13(31.0 \%)$ & $<.001$ \\
\hline Pleural effusion & Yes & $31(32.3 \%)$ & $7(35.0 \%)$ & $11(32.4 \%)$ & $13(31.0 \%)$ & 0.95 \\
\hline Unilateral & Yes & $11(11.5 \%)$ & $3(15.0 \%)$ & $2(5.9 \%)$ & $6(14.3 \%)$ & 0.445 \\
\hline Bilateral & Yes & $20(20.8 \%)$ & $4(20.0 \%)$ & $9(26.5 \%)$ & $7(16.7 \%)$ & 0.575 \\
\hline Mediastinal lymph node swelling & Yes & $31(32.3 \%)$ & $6(30.0 \%)$ & $10(29.4 \%)$ & $15(35.7 \%)$ & 0.818 \\
\hline Hilar lymph node swelling & Yes & $13(13.5 \%)$ & $2(10.0 \%)$ & $6(17.6 \%)$ & $5(11.9 \%)$ & 0.67 \\
\hline Cardiac enlargement & Yes & $16(16.7 \%)$ & $5(25.0 \%)$ & $7(20.6 \%)$ & $4(9.5 \%)$ & 0.232 \\
\hline Pneumothorax & Yes & $2(2.1 \%)$ & $1(5.0 \%)$ & & $1(2.4 \%)$ & 0.455 \\
\hline
\end{tabular}

GGOs: ground-glass opacities

requires hospitalization. In Europe, it was reported that younger age, fewer comorbidities, more extensive radiographic extension and more severe respiratory compromise, and ICU admissions are key features of the clinical presentation of patients with pH1N1 VAP (2). Our study showed similar results, but did not show a significant difference in the severity and rate of respiratory failure from pneumonia among patients with $\mathrm{pH} 1 \mathrm{~N} 1, \mathrm{H} 3 \mathrm{~N} 2$, and B VAP.

Presently, primary viral pneumonia in seasonal influenza is considered to be rare (9), although several groups have reported high rates of primary viral pneumonia in $\mathrm{pH} 1 \mathrm{~N} 1$ VAP. For instance, Perez-Padilla et al. (10) reported primary viral pneumonia in all 18 of their cases (100\%), Champunot et al. (11) in $92 \%$, and Cui et al. (12) in $84 \%$. These differences may be explained by a difference in the affinity of the virus subtypes for the lung and airways. In experimental animal models, pH1N1 virus shows strong replication in the lower airways to alveoli compared with conventional H3N2 (13). No previous studies have compared pH1N1 VAP with H3N2 and B VAP; however, our study showed a higher rate of primary viral pneumonia in patients with pH1N1 VAP than in those with H3N2 or B VAP.

Oliveira et al. (14) investigated the CT findings of seasonal (H3N2, conventional $\mathrm{H} 1 \mathrm{~N} 1$, and B) influenza VAP and reported that bilateral diffuse interstitial/alveolar infiltrates were the most commonly observed radiographic abnormality (9 patients, 52\%), followed by right lower lobe consolidation in $6(35 \%)$ patients. Chest radiographic findings of pH1N1 VAP show that infiltrates are more extensive and frequently interstitial than those of H3N2 VAP (2). It is thus assumed that the CT findings of pH1N1 VAP also differ from those of H3N2 VAP. In previous reports, the common CT findings of pH1N1 VAP include bilateral GGOs and airspace consolidations or combination thereof (15-20). Some authors found no axial or craniocaudal predominance in the distribution in most cases (13), and others found a predominant peribronchovascular and subpleural distribution, resembling organizing pneumonia (OP), particularly in the lower lobes $(18,19)$, or random distribution (20). However, these reports highlighted pH1N1 VAP only and did not compare the CT findings with those of seasonal VAP. Thus, whether these results showed characteristics of pH1N1 VAP has not been elucidated. In the present study, we showed differences in the CT findings among pH1N1, H3N2, and B VAP. The differences included more frequent bilateral GGOs affecting more lobes in patients with pH1N1 VAP versus unilateral GGOs and more frequent consolidation and diffuse bronchial wall thickening in patients with $\mathrm{H} 3 \mathrm{~N} 2$ and $\mathrm{B}$ VAP. These differences appear to reflect the difference in the frequency of mixed bacterial and secondary bacterial pneumonia and in the affinity of the viral subtypes for the airways and lungs.

The histological findings of autopsy cases from past pandemics showed a diffuse alveolar damage (DAD) pattern, however, the major causes of death included bacterial infection (21). In fatal cases of pH1N1 VAP, the most consistent histopathological findings are varying degrees of DAD with hyaline membranes, septal edema, tracheitis, and necrotizing bronchiolitis (22). However, autopsy studies are naturally biased toward the most severe cases. Only one report, by Yeldandi and Colby (23), has described the pulmonary histological findings of patients surviving primary influenza viral pneumonia. They reported the histopathological analysis of 6 sporadic cases of patients with seasonal influenza VAP who underwent lung biopsies. Five patients recovered (2 were treated with corticosteroids for a prior diagnosis of 
OP), and 1 patient died (23). The biopsies showed DAD with hyaline membranes in the non-survivors, but also documented a spectrum of less severe histologic findings with a mild acute lung injury pattern and OP pattern, in addition to confirming previously published pathologic descriptions of influenza pneumonia in non-survivors (21). Our TBLB samples were all obtained from survivors, and the histological findings did not always show DAD, suggesting variety in the severity of histological findings of primary viral pneumonia. A previous report noting the radiopathological findings of pH1N1 VAP in autopsy cases showed that in primary $\mathrm{pH} 1 \mathrm{~N} 1$ viral pneumonia, the areas of airspace consolidation and GGOs correlated with DAD with hyaline membrane formation associated with various degrees of pulmonary congestion, edema, hemorrhage, inflammatory infiltration and bronchiolitis, or OP (24). Our study investigated the histological findings of survivors with both $\mathrm{pH} 1 \mathrm{~N} 1$ and H3N2 VAP, and bilateral GGOs and consolidation correlated with DAD, alveolitis, and OP.

In seasonal influenza VAP, S. pneumoniae (29-48\%) and S. aureus $(7-40 \%)$ have been the most common bacterial isolates causing mixed infections $(1,14,25-28)$. We investigated the pathogens involved in mixed infection with influenza VAP using cultures, urinary antigen tests, and paired sera, and $S$. pneumoniae was found the most frequently, followed by $P$. aeruginosa and S. aureus. Atypical pathogens were found in $11.6 \%$ of cases and should not be ignored. In patients with mixed infection, shadows of the infection of each pathogen superimpose and complicate the CT findings. Careful attention should be paid when physicians or radiologists interpret the CT findings.

In our study, patients with pH1N1 VAP received corticosteroid therapy more frequently than those with $\mathrm{H} 3 \mathrm{~N} 2$ and $\mathrm{B}$ virus infection. The rate of primary viral pneumonia was high in patients with pH1N1 VAP, and some patients were initially diagnosed as having acute interstitial pneumonia or cryptogenic organizing pneumonia, and they received corticosteroid therapy. The diagnosis of these patients was corrected to influenza VAP after confirming positive PCR results or elevated antibody titers. This is the main reason for the higher frequency of corticosteroid therapy in patients with $\mathrm{pH} 1 \mathrm{~N} 1 \mathrm{VAP}$ compared to patients with other subtypes.

There are several limitations associated with this study. The diagnosis of H3N2 influenza VAP was based on paired sera and patients with an acute clinical course and early death may have been missed. The viral subtypes were unknown in 9 patients in whom paired sera could not be obtained due to early deterioration to death and they were thus excluded from the analysis. However, their data may have affected our results. Because this is a nonrandomized observational study, the level of confidence in the results is lessened. A complete diagnostic workup to determine the etiology was not possible in every patient. Finally, this was a single-center study, and the results may not be applicable in other settings.

\section{Conclusion}

The frequency and type of pneumonia differed among three influenza viral subtypes. Of patients with $\mathrm{pH} 1 \mathrm{~N} 1 \mathrm{VAP}$, $80 \%$ developed primary viral pneumonia. The CT findings of pH1N1 VAP differed from those of $\mathrm{H} 3 \mathrm{~N} 2$ or B VAP. Compared with patients with H3N2 or B VAP, those with pH1N1 VAP more frequently showed broad areas of GGOs and less frequently showed consolidation and diffuse bronchial wall thickening. Histologically, the GGOs and consolidation correlated with $\mathrm{DAD}$, acute lung injury, alveolitis, and OP in the survivors. The disease severity and patient mortality did not differ among the three viral subtypes.

\section{The authors state that they have no Conflict of Interest (COI).}

\section{Acknowledgement}

We thank Mina Gochi, Masaki Tamura, Yosuke Miyahara, Shozaburo Yamaguchi, Daido Tokunaga, Hiroo Saito, Kazuyoshi Kurashima, and Tsutomu Yanagisawa at the Department of Respiratory Medicine of Saitama Cardiovascular and Respiratory Center for their detailed and helpful comments regarding the present study.

\section{References}

1. CDC. Past weekly surveillance reports [Internet]. [cited Oct. 10, 2014]. Available from: http://www.cdc.gov/flu/weekly/pastreports.h $\mathrm{tm}$

2. Riquelme R, Torres A, Rioseco ML, et al. Influenza pneumonia: a comparison between seasonal influenza virus and the H1N1 pandemic. Eur Respir J 38: 106-111, 2011.

3. Ishiguro T, Takayanagi N, Yamaguchi S, et al. Etiology and factors contributing to the severity and mortality of communityacquired pneumonia. Intern Med 52: 317-324, 2013.

4. Louria DB, Blumenfeld HL, Ellis JT, Kilbourne ED, Rogers DE. Studies on influenza in the pandemic of 1957-1958. II. Pulmonary complications of influenza. J Clin Invest 3: 213-265, 1959.

5. Mandell LA, Wunderink RG, Anzueto A, et al. Infectious Disease Society of America/American Thoracic Society consensus guidelines on the management of community-acquired pneumonia in adults. Clin Infect Dis 44 (Suppl 2): S27-S72, 2007.

6. Ishiguro T, Takayanagi N, Shimizu Y, Kawabata Y, Yanagisawa T, Sugita Y. Type A influenza pneumonia with diffuse alveolar damage diagnosed by increased antibody titers and immunohistochemical staining. Nihon Kokyuki Gakkai Zasshi 49: 942-948, 2011 (in Japanese, Abstract in English).

7. Ishiguro T, Takayanagi N, Shimizu Y, Kagiyama N, Yanagisawa T, Sugita Y. A patient surviving primary seasonal influenza viral pneumonia: histologic findings obtained via bronchoscopy. Intern Med 52: 2795-2800, 2013.

8. Kobayashi Y, Takayanagi N, Kato E, Ishiguro T, Sugita Y. A patient with influenza pneumonia who developed progressive shortness of breath: differential diagnosis from acute interstitial pneumonia. Kikanshigaku (J. Jpn Soc Resp Endoscopy) 36: 304-308, 2014 (in Japanese, Abstract in English).

9. Treanor JJ. Influenza viruses, including avian influenza and swine influenza. In: Mandell, Douglas, and Bennett's Principles and Practice of Infectious Diseases. 7th ed. Mandell G, Bennett JE, Dolin R, Eds. Churchill Livingstone, Philadelphia, PA, 2010: 2265-2289. 
10. Perez-Padilla R, de la Rosa-Zamboni D, Ponce de Leon S, et al. Pneumonia and respiratory failure from swine-origin influenza A (H1N1) in Mexico. N Engl J Med 361: 680-689, 2009.

11. Champunot $R$, Tanjathan $S$, Kerdsin A, et al. Impact of pandemic influenza (H1N1) virus-associated community-acquired pneumonia among adults in a tertiary hospital in Thailand. Jpn J Infect Dis 63: 251-256, 2010.

12. Cui W, Zhao H, Li X, et al. Factors associated with death in hospitalized pneumonia patients with 2009 H1N1 influenza in Shenyang, China. BMC Infect Dis 10: 145, 2010.

13. Itoh $Y$, Shinya $K$, Kiso $M$, et al. In vitro and in vivo characterization of new swine-origin $\mathrm{H} 1 \mathrm{~N} 1$ influenza viruses. Nature 460: 1021-1025, 2009.

14. Oliveira EC, Marik PE, Colice G. Influenza pneumonia: a descriptive study. Chest 119: 1717-1723, 2001.

15. Amorim VB, Rodrigues RS, Barreto MM, Zanetti G, Hochhegger B, Marchiori E. Influenza A (H1N1) pneumonia: HRCT findings. J Bras Pneumol 39: 323-329, 2013.

16. Ajlan AM, Quiney B, Nicolaou S, Müller N. Swine-origin influenza A (H1N1) viral infection: radiographic and CT findings. AJR Am J Roentgenol 193: 1494-1499, 2009.

17. Agarwal PP, Cinti S, Kazerooni EA. Chest radiographic and CT findings in novel swine-origin influenza A (H1N1) virus (S-OIV) infection. AJR Am J Roentgenol 193: 1488-1493, 2009.

18. Gómez-Gómez A, Magaña-Aquino M, Garcia-Sepúlveda C, et al. Severe pneumonia associated with pandemic (H1N1) 2009 outbreak, San Luis Potosi, Mexico. Emerg Infect Dis 16: 27-34, 2010.

19. Jain S, Kamimoto L, Bramley AM, et al. Hospitalized patients with 2009 H1N1 influenza in the United States, April-June 2009.
N Engl J Med 361: 1935-1944, 2009.

20. Marchiori E, Zanetti G, Hochhegger B, et al. High-resolution computed tomography findings from adult patients with influenza A (H1N1) virus-associated pneumonia. Eur J Radiol 74: 93-98, 2010 .

21. Taubenberger JK, Morens DM. The pathology of influenza virus infections. Annu Rev Pathol 3: 499-522, 2008.

22. Mauad T, Hajjar LA, Callegari GD, et al. Lung pathology in fatal novel human influenza A (H1N1) infection. Am J Respir Crit Care Med 181: 72-79, 2010.

23. Yeldandi AV, Colby TV. Pathologic features of lung biopsy specimens from influenza pneumonia cases. Hum Pathol 25: 47-53, 1994.

24. Marchiori E, Zanetti G, Fontes CA, et al. Influenza A (H1N1) virus-associated pneumonia : high-resolution computed tomography-pathologic correlation. Eur J Radiol 80: e500-e504, 2011.

25. Chang YS, van Hal SJ, Spencer PM, Gosbell IB, Collett PW. Comparison of adult patients hospitalised with pandemic (H1N1) 2009 influenza and seasonal influenza during the "PROTECT" phase of the pandemic response. Med J Aust 192: 90-93, 2010.

26. Scadding JG. Lung changes in influenza. Q J Med 6: 425-465, 1937.

27. Brundage JF. Interactions between influenza and bacterial respiratory pathogens: implications for pandemic preparedness. Lancet Infect Dis 6: 303-312, 2006.

28. Petersdorf RG, Fusco JJ, Harter DH, Albrink WS. Pulmonary infections complicating Asian influenza. AMA Arch Intern Med 103: 262-272, 1959.

(C) 2016 The Japanese Society of Internal Medicine http://www.naika.or.jp/imonline/index.html 